\title{
Comparison of Digital Maps Created by Stereo Plotting and Vectorization Based on Images Acquired by Unmanned Aerial Vehicle
}

\author{
Suk Bae Lee, ${ }^{1 *}$ Taejung Kim, ${ }^{2}$ Young Joon Ahn, ${ }^{3}$ and Jae One Lee ${ }^{4}$ \\ ${ }^{1}$ Department of Civil Engineering, Gyeongnam National University of Science and Technology, \\ 33 Dongjin-ro, Jinju, Gyeongsangnam-do 52725, Republic of Korea \\ ${ }^{2}$ Department of Geoinformatic Engineering, Inha University, \\ 100 Inha-ro, Michuhol-gu, Incheon 22212, Republic of Korea \\ ${ }^{3}$ Department of Geospatial Imagery and Photogrammetry, National Geographic Information Institute, \\ 92 Worldcup-ro, Yeongtong-gu, Suwon, Gyeonggi-do 16517, Republic of Korea \\ ${ }^{4}$ Department of Civil Engineering, Dong-A University, \\ 37, Nakdong-saero 550 beon-gil, Saha-gu, Busan 49315, Republic of Korea
}

(Received August 12, 2019; accepted September 27, 2019)

Keywords: UAV, point cloud data, DSM, digital mapping, stereo plotting

Unmanned aerial vehicles (UAVs) are becoming an economical and efficient means to create digital maps, but until now, the vectorization method has been used to extract topographic features from orthoimages for large-scale mapping. The aim of this study is to determine the potential of UAV photogrammetry for digital mapping with stereo plotting, which can include vertical position information. In this study, the entire work process of UAV photogrammetry was performed using a case study in Korea, where 314 images were acquired with a BirdsEyeView Aerobotics FireFly6 PRO. To compare the results, digital maps were created by stereo plotting and vectorization. To evaluate the accuracy of the digital maps created by stereo plotting, we evaluated the accuracy of the aerial triangulation (AT) work from ground control points (GCPs) and the position accuracy of the maps from checkpoints (CPs). The accuracy of the AT work was on the sub-centimeter order and the position accuracy of the maps was on the sub-decimeter order. We also compared a digital map produced by the Korean National Geographic Information Institute (NGII) with the maps created by stereo plotting and vectorization to evaluate the mapping accuracy. From the results of the comparison, we found that the maps created by stereo plotting were more accurate than those created by vectorization.

\section{Introduction}

Unmanned aerial vehicle (UAV) photogrammetry is a photogrammetric measurement platform that operates with remote control semiautonomously or autonomously without a pilot. The platform is equipped with a photogrammetric measurement system, including, but not limited to, a small or medium-size still-video or video camera, a thermal or infrared camera

*Corresponding author: e-mail: sblee@gntech.ac.kr https://doi.org/10.18494/SAM.2019.2553 
system, an airborne LiDAR system, or a combination thereof. Current standard UAVs allow the registration and tracking of the position and orientation of the implemented sensors in a local or global coordinate system. UAV photogrammetry opens various new applications in the closerange domain, combining aerial and terrestrial photogrammetries, and also introduces new realtime applications and low-cost alternatives to classical manned aerial photogrammetry. ${ }^{(1-3)}$

For photogrammetric applications, a UAV system is generally composed of a camera, a global navigation satellite system (GNSS), and an inertial measurement unit (IMU). The camera takes overlapping images over the area covered while the UAV is flying. The GNSS is responsible for the georeferencing of the images. To carry out the process of georeferencing, the image processing software has to know the real-world GNSS coordinates of a small number of visibly identifiable locations in the collected aerial imagery. ${ }^{(4)}$

In 2012, Rosenell and Honkavaara published a paper on the automatic generation of high-quality, dense point clouds from digital images by image matching as a cutting-edge advancement in digital photogrammetric technology. ${ }^{(5)}$ UAVs are becoming an economical and efficient means to create digital maps and also construct a digital twin of the topography for a small area because a UAV can obtain high-resolution images rapidly, conveniently, and economically as compared with conventional manned aerial photogrammetry. The possibility of large-scale mapping using UAV photogrammetry has already been proven in many studies. ${ }^{(6-11)}$ However, until now, the vectorization method has been used for large-scale mapping by extracting topographic features as a layer in orthoimages. ${ }^{(12-15)}$ It is difficult to consider such mapping as genuine digital mapping because it cannot obtain 3D height information. The height information of required points can be obtained using a digital surface model (DSM) or a digital elevation model (DEM) created through point cloud data in UAV photogrammetry. However, to create a 3D digital map, both the horizontal position information and the vertical position information should be included, where the latter should be displayed in the form of contour lines. Therefore, in mapping using UAV photogrammetry, the stereo plotting method should accurately extract such vertical position information of the topographic features similarly to that in the case of traditional aerial photogrammetry. However, in UAV photogrammetry, stereo plotting is difficult because a huge number of images are obtained compared with that in traditional aerial photogrammetry. In addition, commercial UAV software does not support stereo plotting functions. Traditional aerial photogrammetry has been used in many countries to produce topographic maps of the countries. In Korea, digital topographic maps in three scales, 1:5000, 1:25000, and 1:50000, have been produced as national base maps using aerial photogrammetry.

In this study, we attempted to determine whether map creation by stereo plotting in UAV photogrammetry is possible. Therefore, the purpose of this study was to investigate whether UAV photogrammetry can create large-scale digital topographic maps by the stereo plotting method. For this purpose, a study area was selected and UAV photogrammetry and a ground control point (GCP) survey were carried out. The overall workflow of this study is shown in Fig. 1.

As shown in Fig. 1, the work plan was first set up and a field investigation was conducted in the study area. Appropriate positions of the GCPs were chosen in the field, air photo signals were installed, and a GCP survey was carried out. Then, images were acquired over the 


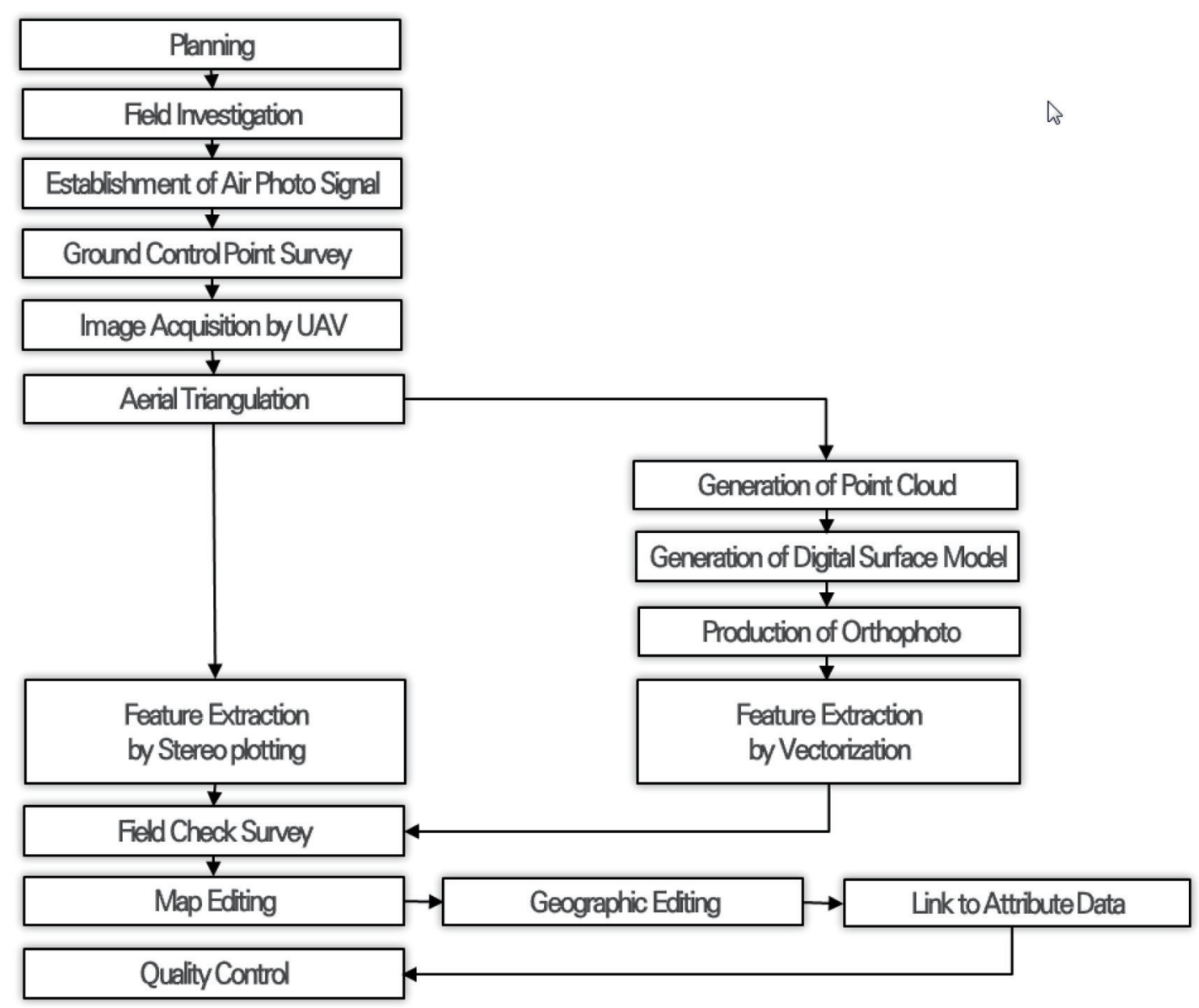

Fig. 1. Overall work flowchart of UAV photogrammetry adopted in this study.

study area using a UAV with a Sony a6000 digital camera, and aerial triangulation (AT) was performed. These images were processed through a photogrammetric workflow to obtain a point cloud or a DSM, an orthophoto of the scene using commercial UAV software. Drawing topographic features, which is the most important part of this study, was carried out in two ways. One was using the stereo plotting method as in traditional aerial photogrammetry and the other was using the vectorization method. Two types of digital map created by the two methods were edited through a field check survey and map editing. These maps were edited into final digital maps with geographic editing and linked to attribute data processes. Finally, the two topographic maps were compared and evaluated to distinguish differences for quality control.

\section{Materials and Methods}

The Korean National Geographic Information Institute (NGII) published the "Guidelines for Public Survey Using UAV" as NGII Notice No. 2018-1075 in March 2018. ${ }^{(16)}$ These guidelines specify standard work processes and inspection standards to encourage the use of 
UAVs in public surveys in Korea. Thus, the creation of the digital maps and the evaluation of the UAV performance were carried out in accordance with this guideline and "Regulation for

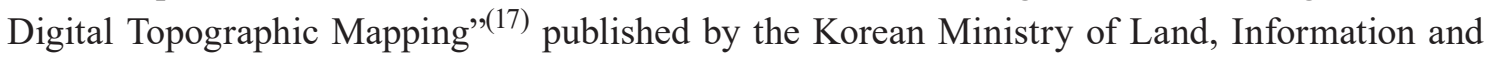
Transportation (MOLIT) as Order No. 209.

\subsection{Planning and field investigation}

The planning step requires activities such as UAV selection, software selection, the detailed analysis of the study area, decisions on the ground sample distance (GSD), flight height, and overlapping ratio. This study focused on the capability of 3D stereo plotting of the images acquired by a UAV. Thus, the study area was chosen to be an urban area because urban areas have many topographic features such as houses, buildings, roads, and trees. The downtown area of Incheon City, Korea, with a size of $0.5 \mathrm{~km}^{2}$ was selected as the study area, as shown in Fig. 2. This area corresponds to two Korean 1:1000-scale digital maps with sheet numbers 37610449 and 37610450.

Following the planning step, a field investigation was conducted after the selection of the study area. In this field investigation, a safe place for UAV landing was chosen, and appropriate locations for the GCPs and checkpoints (CPs) were investigated. In addition, obstacles such as poles and electricity transmission towers in and around the study area were investigated and the possibility of UAV flight around the obstacles was verified.

\subsection{Establishment of air photo signal and GCP survey}

According to Article 9 of the Guideline for Public Survey Using UAV, the GCPs should be evenly placed in consideration of the shape of the work area, the course direction, and so forth. ${ }^{(16)}$ Also, these GCP locations should be at points that can be clearly distinguished in the image

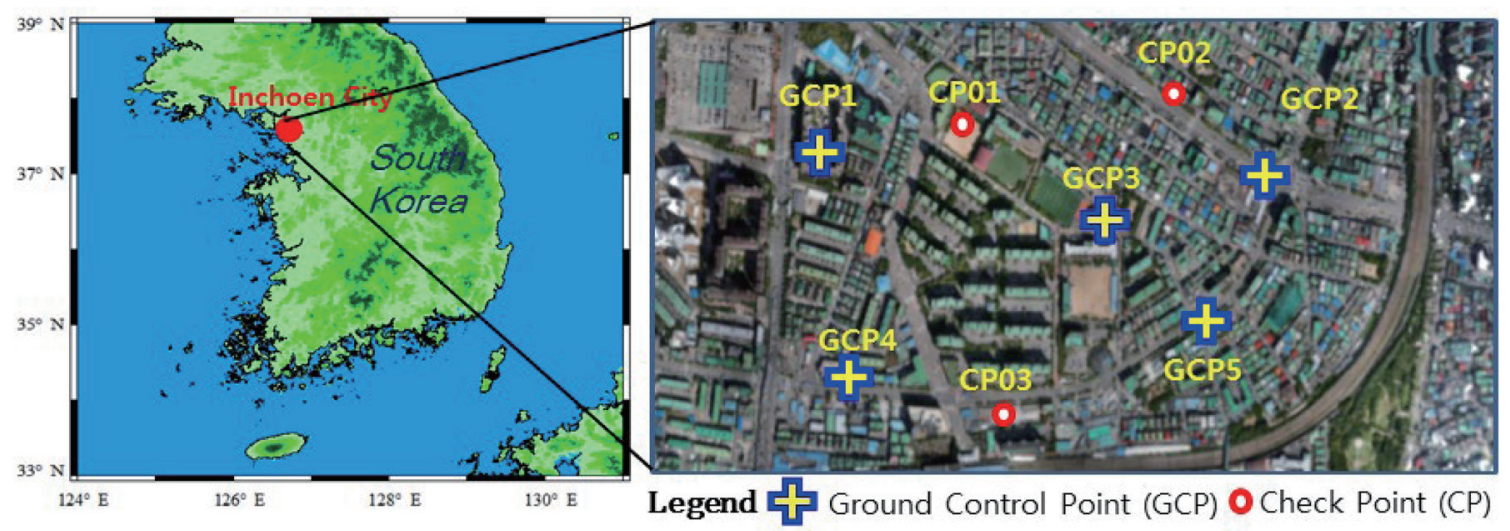

(a)

(b)

Fig. 2. (Color online) Photographs of (a) location map of the study area in Korea and (b) location map of GCPs and CPs. 
and should be at least nine GCPs per $1 \mathrm{~km}^{2}$. According to Article 11, the number of CPs must be at least one-third of that of GCPs, and a sufficient number must be ensured according to the difficulty of the work. However, if there are fewer CPs, there must be three CPs. Therefore, we decided to set the number of GCPs to five and that of CPs to three because the study area was $0.5 \mathrm{~km}^{2}$. Then, an air photo signal, as shown in Fig. 3(a), should be installed at these eight points. However, if it is difficult to install the air photo signal in a downtown area, points that can be clearly identified in the image can be selected as the GCP locations, as shown in Fig. 3(b). In Article 10, horizontal and vertical control survey methods are defined. Therefore, according to Article 10, a GNSS network-RTK survey was performed for horizontal positioning [Fig. 4(a)] and a level survey was performed for vertical positioning [Fig. 4(b)]. As a result of these GCP surveys, transverse mercator (TM) horizontal coordinates and heights above the Korean mean sea level of the five GCPs and three CPs were acquired.

\subsection{Image acquisition and image processing}

In August 2018, UAV flights were operated over the study area using a Firefly6 PRO, which adopted vertical take-off and landing (VTOL) for image acquisition. The planned flight height was $217 \mathrm{~m}$ to obtain a GSD of $5 \mathrm{~cm}$, sufficient to make a 1:1000-scale digital map. A total

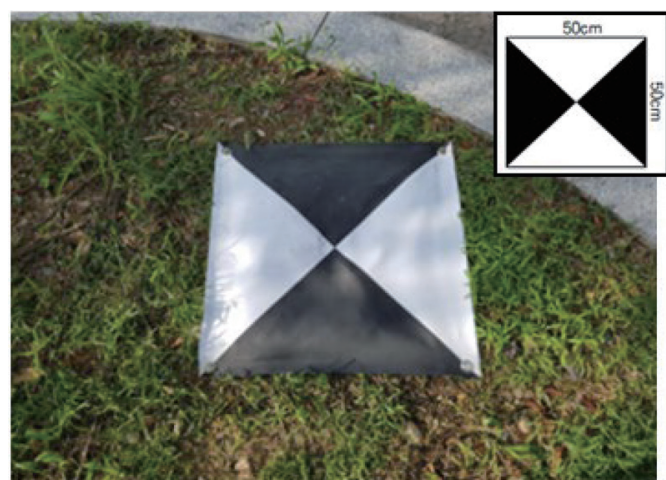

(a)

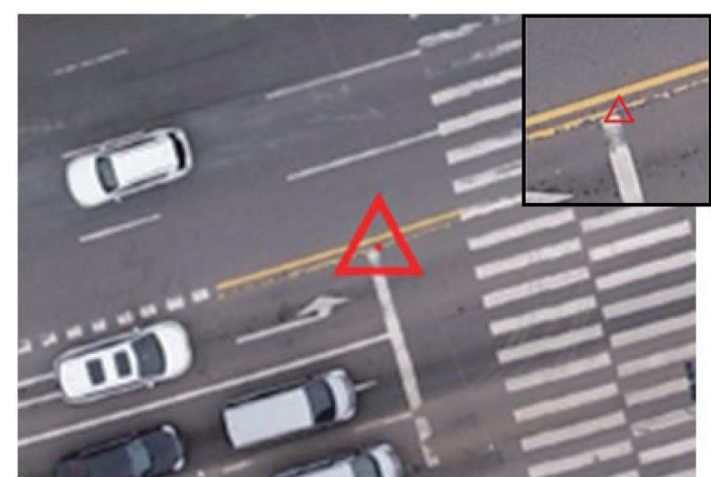

(b)

Fig. 3. (Color online) Photographs of (a) installation of air photo signal on the ground and (b) replacement of air photo signal in the road.

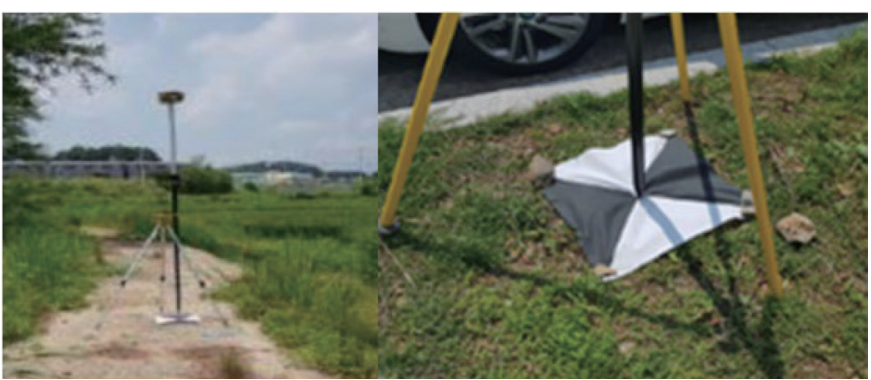

(a)

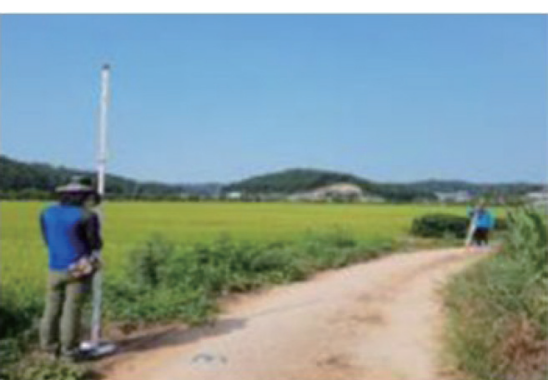

(b)

Fig. 4. (Color online) Photographs of (a) horizontal GNSS Network-RTK survey and (b) vertical level survey. 
of 314 digital images with $85 \%$ forward and $80 \%$ side overlaps according to Article 13 of the Guideline for the Public Survey Using UAV were acquired with a Sony a6000 camera. Figure 5 shows the (a) Firefly6 PRO UAV and (b) Sony a6000 camera.

Following the image acquisition step, image processing was carried out. According to Article 17 of the Guideline for the Public Survey Using UAV, AT should be performed by automatic matching and must use software that has the function of bundle block adjustment (BBA) and its equivalent function. ${ }^{(16)}$ AT is the process of contiguous densifying and extending ground control through computational means. This operation includes establishing GCPs, performing interior orientation (IO) manually, measuring and transferring all tie, check, and control points appearing on all photographs, and performing a least-squares block adjustment. This process ultimately provides exterior orientation (EO) parameters for photographs and 3D coordinates for the measured object points. The purpose of AT is to refine the EO parameters computed by direct georeferencing for each image, which will help to achieve the desired accuracy while generating the DEM. ${ }^{(15)}$ The extracted common features visible in multiple images are needed as tie points for image orientation. The selection of homologous points in the images was performed automatically using AgiSoft PhotoScan software. The mathematical relationship between images, camera geometry, and ground space is computed during block triangulation, which is commonly combined with an error minimization strategy and is then referred to as BBA. $^{(12)}$ For AT, the five GCP coordinates were entered in this software as known control point coordinates. Table 1 shows the details of the image acquisition and processing performed in this study.

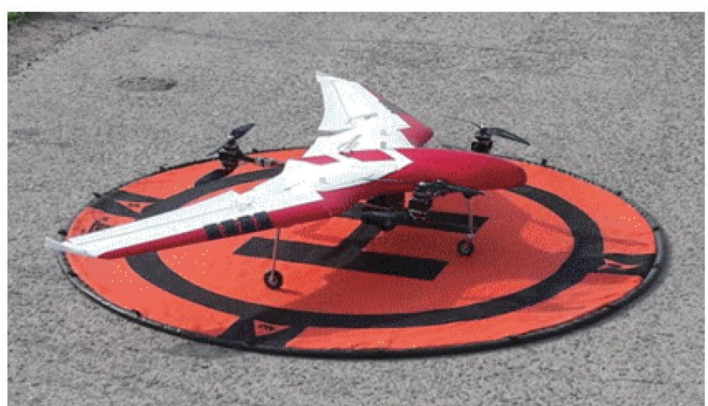

(a)

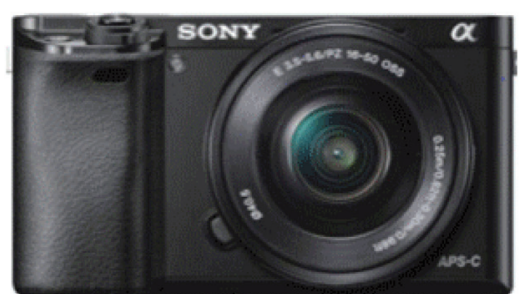

(b)

Fig. 5. (Color online) Photographs of (a) FireFly6 Pro and (b) Sony a6000 camera.

Table 1

Image acquisition and image processing.

\begin{tabular}{lc}
\hline No. of images & 314 \\
Flying altitude (average) & $219 \mathrm{~m}$ \\
GSD & $3.9 \mathrm{~cm} / \mathrm{pixel}$ \\
Coverage area & $0.878 \mathrm{~km}^{2}$ \\
No. of GCPs & 5 \\
No. of CPs & 3 \\
No. of tie points & 219223 \\
\hline Camera model & Resolution: $6000 \times 4000$ \\
(ILCE-6000) & Focal length: $20 \mathrm{~mm}$ \\
& Pixel size: $4 \times 4 \mu \mathrm{m}^{2}$ \\
\hline
\end{tabular}




\subsection{Generation of DSM and orthophoto}

After AT, a DSM and an orthophoto in the study area were generated using AgiSoft PhotoScan software, as shown in Fig. 6. The process includes 1) uploading the aerial images acquired by a UAV with a camera (add photos), 2) matching images using tie points, which are selected automatically (align photos), 3) creating an approximate 3D model using the point cloud generated in the previous step (build mesh), 4) marking GCP locations on the image and entering the GCP coordinates, 5) generating a point cloud (build dense cloud), 6) generating the DSM (build mesh), 6) generating the orthophoto (build texture), and 7) generating the mosaic orthophoto and 3D model. Figure 7 shows an image sample and an enlargement of the dotted circle area. Figure 8 shows the generated (a) orthophoto and (b) DSM of the study area.

\subsection{Digital mapping by both stereo plotting and vectorization}

In Korea, Regulation for Digital Topographic Mapping published by MOLIT ${ }^{(17)}$ sets the standard work process and inspection standard for digital map production. In this regulation,

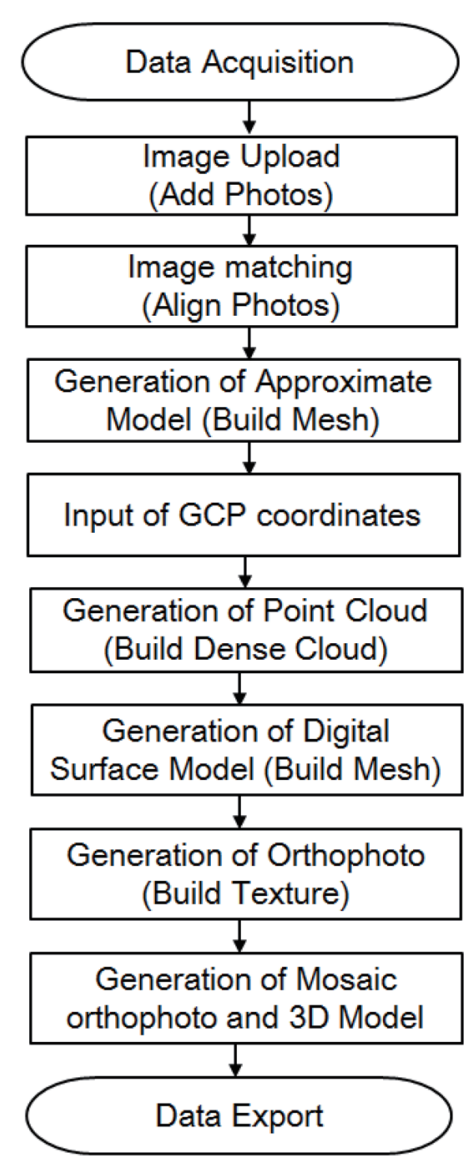

Fig. 6. Flowchart of data processing from image to output using PhotoScan software in UAV photogrammetry. 


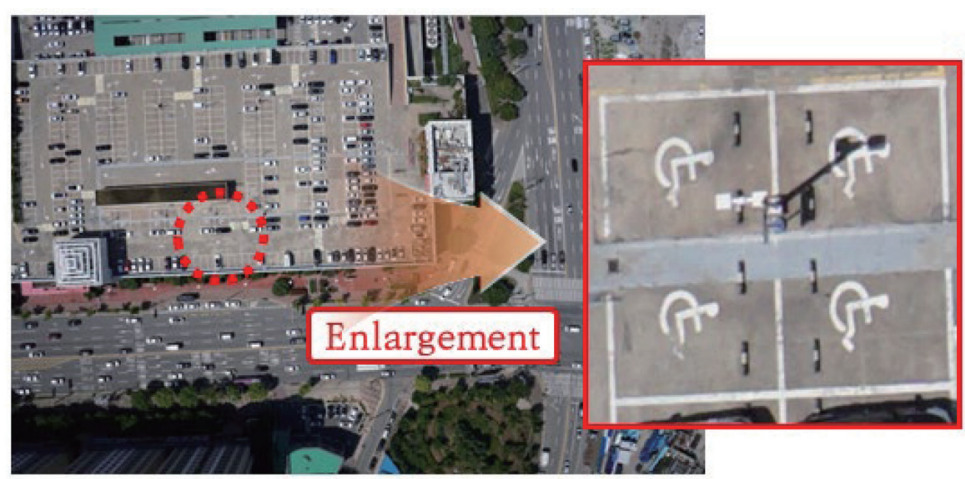

Fig. 7. (Color online) Image sample acquired by Firefly6 Pro with Sony a6000 camera.

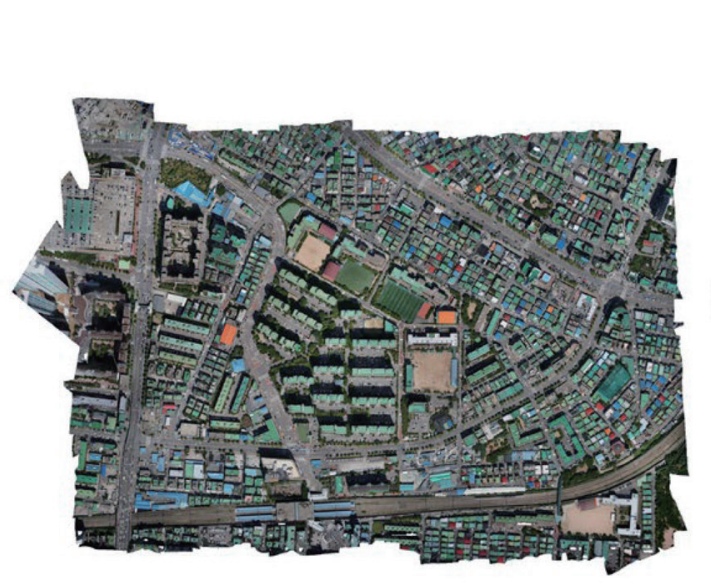

(a) (b)

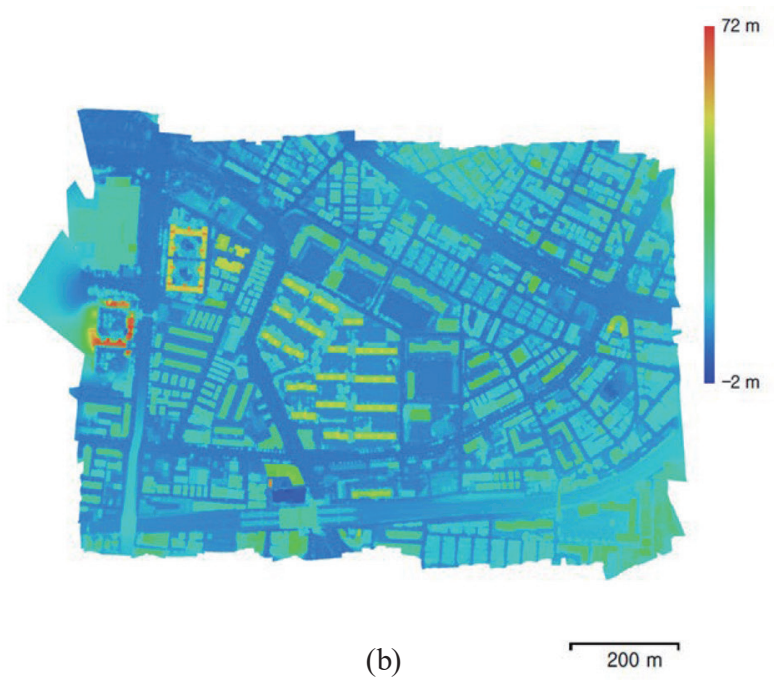

Fig. 8. (Color online) Generated (a) mosaic orthophoto and (b) DSM of the study area.

a digital topographic map is defined to contain various pieces of spatial information such as location on Earth's surface as well as geographic features and place names that are displayed by symbols, letters, and attributes according to a certain scale.

In this study, the digital mapping of the study area was carried out using both the stereo plotting and vectorization methods. In the stereo plotting method, the height (i.e., the vertical position information) is determined from stereo photographs using a stereo plotter. The stereo plotter requires two photographs that show a considerable overlap and are corrected for distortion due to photo angles. The photos are placed on transparent media and projected using a light source, so that they overlap. The operator, using a special set of optics, then sees the image as three-dimensional owing to the differing perspective of each photo. The optics of the stereo plotter allow the operator to plot the contours and features. In this stereo plotting step, a Geo3DI stereo plotter was used, and an operator with more than 10 years of experience used the device. Maps were created by stereo plotting according to the procedures of Regulation 
for Digital Topographic Mapping. In the rule, the objects of restitution include roads, water systems, railways, buildings, facilities, contour lines, and heights. Also, maps are created by vectorization by the same operator according to the same rule. Figure 9 shows (a) a map created by stereo plotting and (b) the operator working with the Geo3DI stereo plotter.

\subsection{Map editing}

According to the Korean Regulation for Digital Topographic Mapping, for digital maps after feature extraction, map editing should be performed. This step is composed of geographic editing and linking to attribute data. Map editing means the operation of editing or supplementing drawing or map input data using the data obtained from a field check survey. Geographic editing refers to the work of supplementing and editing a map using the data of the topographic features confirmed by a field check survey to ensure the compatibility of the data, a standard code (graphic code, layer code), and a standard sequence (type, size, preference, structure, and direction of a figure that constitutes a digital topographic map) to complete a digital topographic map and input the administrative boundaries, place names, and annotations. The link to attribute data refers to the work of constructing the geometry of the terrain and objects to understand the geographical correlations between the features and inputting objectspecific attributes. Both geographic editing and linking to attribute data were performed using the two digital topographic maps produced by stereo plotting and vectorization. Figure 10 shows the results.

Figure 10 shows (a) a stereo-plotted map overlapped on a mosaic orthophoto, (b) a map with geographic editing based on the stereo-plotted map, (c) a map with linking to attribute data based on the map in (b), (d) a vectorization map overlapped on a mosaic orthophoto, (e) a map with geographic editing based on the vectorization map, and (f) a map with link to attribute data based on the map in (e).

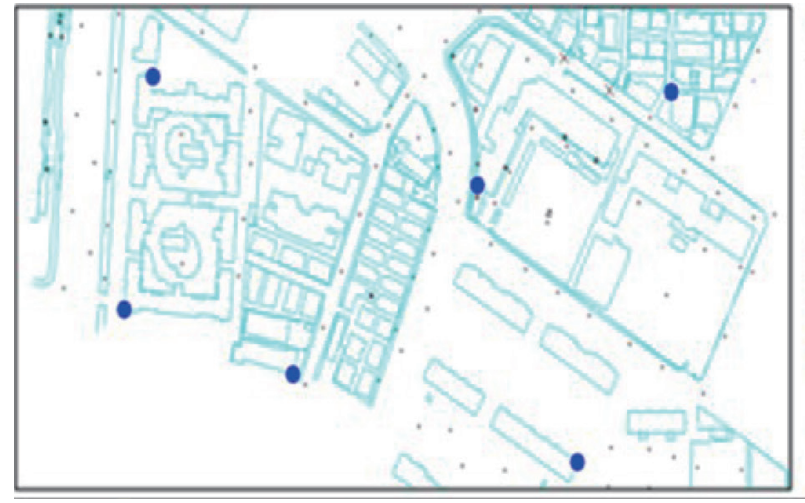

(a)

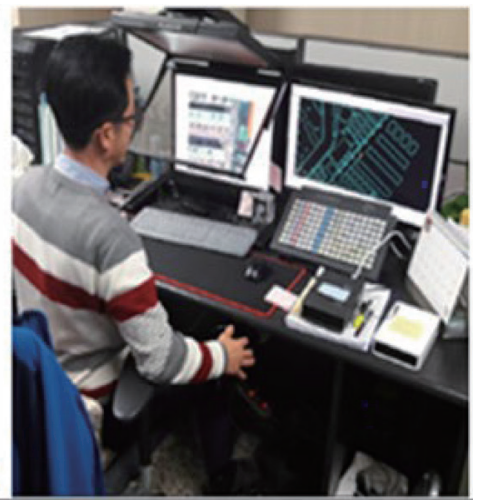

(b)

Fig. 9. (Color online) Photographs of (a) map created by stereo plotting and (b) operator working with Geo3DI stereo plotter. 


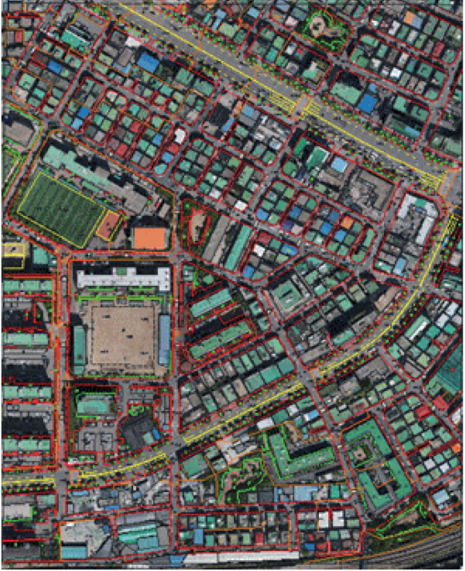

(a)

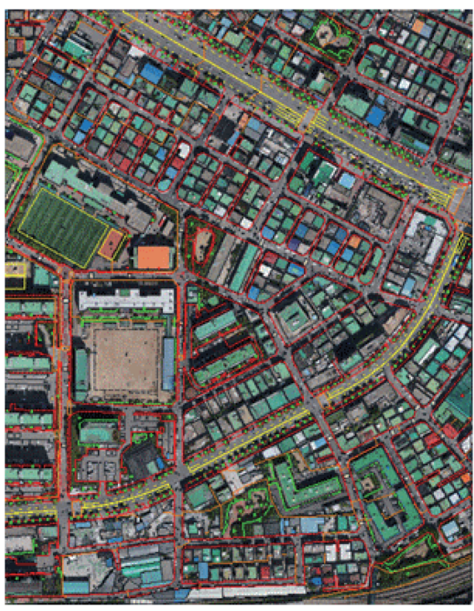

(d)

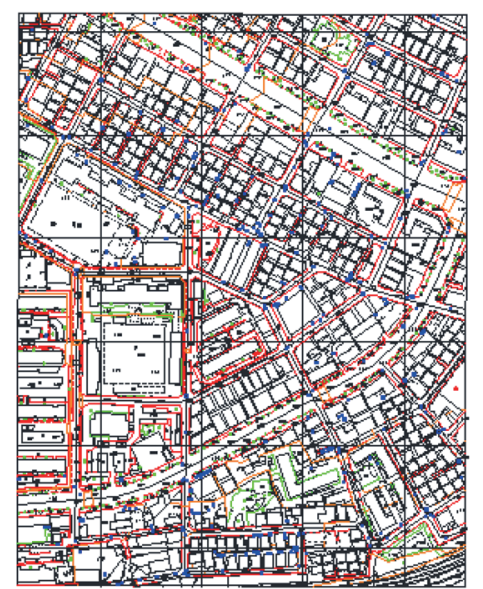

(b)

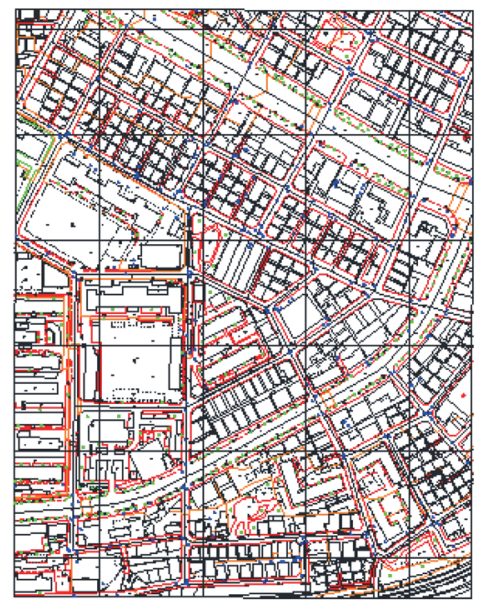

(e)

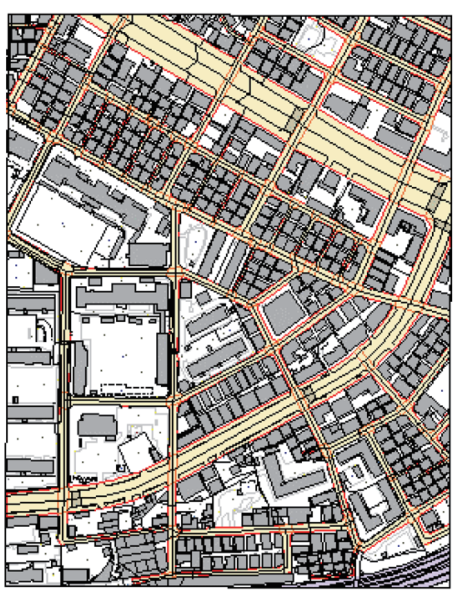

(c)

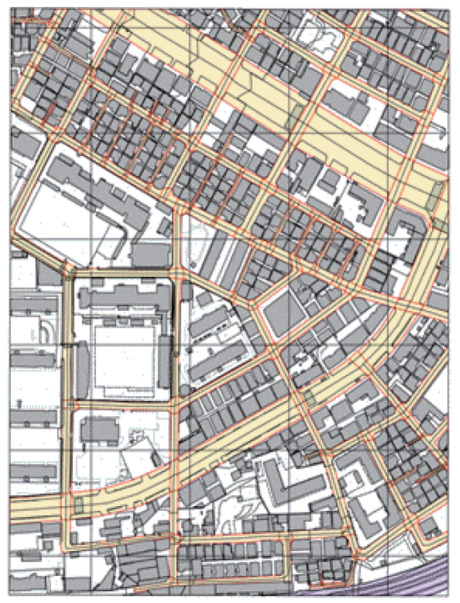

(f)

Fig. 10. (Color) Photographs of (a) a stereo-plotted map overlapped on a mosaic orthophoto, (b) a map with geographic editing based on the stereo-plotted map, (c) a map with link to attribute data based on map (b), (d) a vectorization map overlapped on a mosaic orthophoto, (e) a map with geographic editing based on the vectorization map, and (f) a map with link to attribute data based on map (e).

\subsection{Quality control}

The final step is quality control, as shown in Fig. 1; thus, accuracy assessment was performed in AT and the 3D stereoscopic model. For accuracy assessment in AT, the root mean square error (RMSE) of the GCPs was calculated using the differences between the GCP coordinates obtained from the GCP survey and those automatically obtained from the mosaic orthophoto using PhotoScan software. The results were displayed in the Agisoft PhotoScan processing report file. Table 2 shows the results. An RMSE of $\pm 1.251 \mathrm{~cm}$ for horizontal position accuracy and an RMSE of $\pm 0.527 \mathrm{~cm}$ for vertical position accuracy were achieved from the assessment. According to Article 17 of the Guideline for Public Survey Using UAV, the accuracy of the horizontal position and the calculated height are required to comply 
Table 2

RMSE for accuracy assessment in AT at GCPs (unit: cm).

\begin{tabular}{lcccc}
\hline Numbers & $\Delta X$ & $\Delta Y$ & $\Delta X Y$ & $\Delta Z$ \\
5 & 0.878 & 0.889 & 1.251 & 0.527
\end{tabular}

Table 3

Statistics of the difference between GNSS coordinates and map coordinates at CPs (unit: $\mathrm{m}$ ).

\begin{tabular}{lccccc}
\hline \multirow{2}{*}{ Division } & \multicolumn{3}{c}{ Horizontal differences } & & Height differences \\
\cline { 2 - 4 } & $\Delta X$ & $\Delta Y$ & $\Delta X Y$ & & $\Delta Z$ \\
\hline Minimum & 0.028 & 0.009 & 0.034 & & 0.048 \\
Maximum & 0.075 & 0.065 & 0.077 & & 0.124 \\
Mean & 0.045 & 0.031 & 0.061 & & 0.074 \\
RMSE & \pm 0.066 & \pm 0.039 & \pm 0.064 & & \pm 0.082 \\
\hline
\end{tabular}

Table 4

Korean standard on feature extraction by stereo plotting (unit: $\mathrm{m}$ ).

\begin{tabular}{|c|c|c|c|c|c|c|c|}
\hline \multirow[b]{2}{*}{ Output scale } & \multirow[b]{2}{*}{ GSD } & \multicolumn{3}{|c|}{ RMSE } & \multicolumn{3}{|c|}{ Maximum error } \\
\hline & & $\begin{array}{c}\text { Horizontal } \\
\text { position }\end{array}$ & Contour line & Height point & $\begin{array}{c}\text { Horizontal } \\
\text { position }\end{array}$ & Contour line & Height point \\
\hline $1 / 1000$ & 0.12 & \pm 0.2 & \pm 0.3 & \pm 0.15 & 0.4 & 0.6 & 0.3 \\
\hline
\end{tabular}

with the Aerial Photogrammetry Regulation ${ }^{(18)}$ standard. Thus, in Article 56 of the Aerial Photogrammetry Regulation, ${ }^{(18)}$ the limit of the adjustment calculations and errors is defined as an RMSE of $0.2 \mathrm{~m}$ and a maximum of $0.4 \mathrm{~m}$ when the scale is 1:1000 to 1:1200. Thus, the results shown in Table 2 satisfy these Korean standards. This means that AT using PhotoScan software was performed very well.

After AT, the 3D stereoscopic model was set up. From the stereoscopic model, three CPs were digitized for accuracy assessment. Table 3 shows the results of accuracy assessment using the residuals at CPs. In this study, an RMSE of $\pm 0.064 \mathrm{~m}$ for horizontal position accuracy and an RMSE of $\pm 0.082 \mathrm{~m}$ for height accuracy were achieved from the assessment. The required accuracy of feature extraction by stereo plotting according to Article 67 of the Aerial Photogrammetry Regulation is shown in Table 4. The results shown in Table 3 can be seen to satisfy the criteria shown in Table 4. This means that stereo plotting was carried out satisfactorily.

\section{Results and Discussion}

The entire process of UAV photogrammetry, which was discussed in Sect. 2, was performed in this study. We attempted map creation by stereo plotting using a Geo3DI stereo plotter, as with traditional aerial photogrammetry. The operator, however, suffered from dizziness in the process of using stereoscopic vision. This was because the shooting angle of the UAV images deviated considerably from the vertical and there was significant vertical exaggeration. However, over time, the operator became accustomed to the process and could create a map using stereoscopic vision. Therefore, it is possible to produce a 1:1000-scale map as a national topographic map using UAV photogrammetry. 
Table 5

Statistics of the difference between NGII map coordinates and map coordinates obtained by two methods at seven control points (unit: $\mathrm{m}$ ).

\begin{tabular}{|c|c|c|c|c|c|c|}
\hline \multirow{2}{*}{ Division } & \multicolumn{3}{|c|}{ Stereo plotting } & \multicolumn{3}{|c|}{ Vectorization } \\
\hline & $\Delta X$ & $\Delta Y$ & $\Delta X Y$ & $\Delta X$ & $\Delta Y$ & $\Delta X Y$ \\
\hline Minimum & 0.004 & 0.010 & 0.037 & 0.008 & 0.008 & 0.037 \\
\hline Maximum & 0.138 & -0.115 & 0.156 & 0.160 & -0.137 & 0.162 \\
\hline Mean & 0.050 & 0.059 & 0.085 & 0.059 & 0.066 & 0.101 \\
\hline RMSE & \pm 0.066 & \pm 0.066 & \pm 0.094 & \pm 0.075 & \pm 0.080 & \pm 0.110 \\
\hline
\end{tabular}

From the accuracy assessment of the AT work and digital mapping by stereo plotting, it was found that the quality of the map was very good. A comparison of topographic maps produced by stereo plotting and vectorization is shown in Fig. 10 to be very simple. Therefore, to quantitatively compare the digital maps produced by the two methods, we used the 1:1000-scale digital topographic map produced by the NGII in 2017. The map was downloaded from the "Land Information Platform" managed by the NGII. ${ }^{(19)}$ For comparison, seven control points such as buildings, playgrounds, parking lots, and roads shown in the digital topographic map were selected and the coordinates of these seven points were digitized in the digital map produced by the NGII. In addition, we calculated the difference by comparing the horizontal coordinates on the NGII digital topographic map by digitizing the horizontal coordinates for the same seven points on the digital map produced by stereo plotting and vectorization. The resulting statistics are shown in Table 5. Since the digital map created by vectorization is not a 3D topographic map owing to the lack of height information, only the horizontal coordinates are compared.

As shown in Table 5, the mean of the difference from the digital map produced by stereo plotting was $0.085 \mathrm{~m}$ and the RMSE was $\pm 0.094 \mathrm{~m}$. Also, the difference from the digital map produced by vectorization was $0.101 \mathrm{~m}$ and the RMSE was $\pm 0.110 \mathrm{~m}$. Therefore, the residual of the digital map created by stereo plotting was smaller than that of the digital map created by vectorization. This means that digital mapping by stereo plotting is more accurate than that by vectorization.

\section{Conclusions}

In this study, we compared digital maps created by stereo plotting and vectorization using the images acquired by a UAV. The whole work process of UAV photogrammetry consisted of planning, field investigation, a GCP survey, image acquisition by a UAV, AT, map creation by stereo plotting and vectorization, a field check survey, map editing, and quality control. It was performed using a case study in Korea, where 314 images were acquired with a BirdsEyeView Aerobotics FireFly6 PRO. The creation of digital maps and the evaluation of UAV achievements were carried out in accordance with the Guidelines for Public Survey Using UAV because these guidelines specify standard work processes and inspection standards in Korea. In this study, it was confirmed that a digital map can also be produced by stereo plotting using a conventional stereoplotter in UAV photogrammetry.

The accuracy of the map created by stereo plotting was evaluated using the difference between the GNSS coordinates and the 3D stereoscopic model's coordinates. The horizontal 
position had an RMSE of $\pm 0.064 \mathrm{~m}$ and the vertical position had an RMSE of $\pm 0.082 \mathrm{~m}$. These values satisfy the digital mapping standard of Korea. In addition, we calculated the difference between the horizontal coordinates of the NGII digital topographic map and those of the digital maps created by stereo plotting and vectorization at seven control points. From this evaluation, it was confirmed that the digital map created by stereo plotting is more accurate than that created by vectorization.

To construct spatial information by UAV photogrammetry, the quality of the data acquired through the sensor is important. Currently, there are no accurate calibration data for UAV sensors, and the accuracy depends on the self-calibration method embedded in UAV commercial software. However, precise camera calibration data are required to perform AT accurately. Therefore, it is necessary to develop a precise sensor calibration technique in the near future to increase the utilization of UAVs.

\section{Acknowledgments}

This work was supported by the National Geographical Information Institute in Korea as a 2018 research project named "Technology development and cost estimation research on the construction of spatial information using UAV".

\section{References}

1 H. Eisenbeiß: UAV Photogrammetry, DISS ETH NO. 18515, Ph.D. Dissertation, University of Technology Dresden, Germany (2009).

2 F. Neitzel and J. Klonowski: Int. Arch. Photogramm. Remote Sens. Spatial Inf. Sci. 38 (1/C22) (2011) 39.

3 G. J. Grenzdorffer, A. Engel, and B. Teichert: Int. Arch. Photogramm. Remote Sens. Spatial Inf. Sci. 37 (2008) 1207.

4 K. Pothuganti, M. Jariso, and P. Kale: Int. J. Innovative Res. Comput. Commun. Eng. 5 (2017) 1170. https://doi. org/10.15680/ijircce.2017.0501126

5 T. Rosenell and E. Honkavaara: Sensors 12 (2012) 453. https://doi:10.3990/s120100453

6 M. J. Ahmad, A. Ahmad, and K. D. Kanniah: 2018 IOP Conf. Series: Earth Environ. Sci. 169 (2018) 012077.

7 W. S. Udin and A. Ahmad: Int. J. Sci. Eng. Res. 3 (2012)

8 X. Tianyun, T. Xiaocheng, Y. Defang, X. Yonghe, and Y. Hongliang: Int. J. Control Autom. 8 (2015) 279. http://dx.doi.org/10.14257/ijca.2015.8.3.28

9 A. Ahmad: Pertanika J. Sci. Technol. 19 (2011) 51.

10 H. Woo, S. Back, and W. Hong: Spatial Inf. Res. 26 (2018) 639. https://doi.org/10.1007/s41324-018-0208-9

11 I. Colomina and P. Molina: ISPRS J. Photogramm. Remote Sens. 92 (2014) 79. https://dx.doi.org/10.1016/ j.isprsjprs.2014.02.013

12 M. Koeva, M. Muneza, C. Gevaert, M. Gerke, and F. Nex: Survey Rev. 50 (2018) 361. https://doi:10.1080/0039 6265.2016 .1268756

13 S. B. Lee, Y. J. Ahn, and J. K. Noh: Proc. 21st Int. Symp. Geospatial Information Science and Urban Planning (GISUP, 2019) 143.

14 J. Lee and S. Sung: Spat. Inf. Res. 24 (2016) 141. https://doi.org/10.1007/s41324-016-0015-0

15 Aerial Triangulation: https://www.satpalda.com/ (accessed May 2019)

16 National Geographical Information Institute: Guidelines for the Public Survey Using UAV. NGII Notice No. 2018-1075 (2018)

17 Ministry of Land, Information and Transportation: Regulations for the Digital Topographic Mapping. MOLIT Order No. 209 (2015)

18 National Geographical Information Institute: Aerial Photogrammetry Regulations. NGII Notice No. 20162609 (2016).

19 Land Information Platform: http://map.ngii.go.kr/mn/ (accessed November 2018) 


\section{About the Authors}

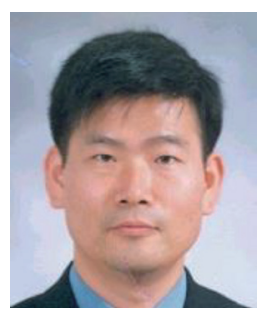

Suk Bae Lee received his B.E., M.S., and Ph.D. degrees from Sungkyunkwan University, Korea, in 1986, 1989, and 1997, respectively. From 1997 to 2003, he worked as a professor at Vision College of Jeonju. Since 2004, he has been a professor at Gyeongnam National University of Science and Technology, Korea. He was the president of the Korean Society for Geospatial Information Science in 2018. His research interests are in geoid modeling, GNSS application, and UAV photogrammetry. (sblee@gntech.ac.kr)

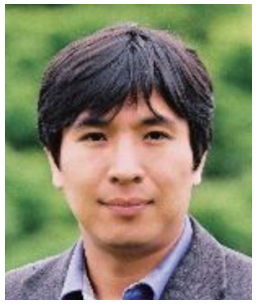

Taejung Kim received his B.S. degree from Korea Advanced Institute of Science and Technology (KAIST), Korea, in 1991 and his M.S. and Ph.D. degrees from University College London, UK, in 1992 and 1996, respectively. From 1995 to 2003, he was a researcher at KAIST, Korea. Since 2003, he has been a professor at Inha University, Korea. His research interests are in remote sensing, digital photogrammetry, and computer vision.

(tezid@inha.ac.kr)

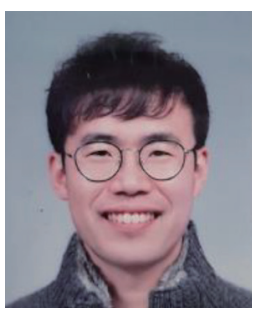

Young Joon Ahn received his B.S. and M.S. degrees from Sungkyunkwan University, Korea, in 1999 and 2002, respectively. He is currently a Ph.D. student at Sungkyunkwan University. Since 2006, he has been a public officer at NGII (National Geographical Information Institute), the Ministry of Land, Infrastructure and Transport, Korea. His research interests are in photogrammetry, geoid modeling, GNSS, and UAV survey.

(4mylove@korea.kr)

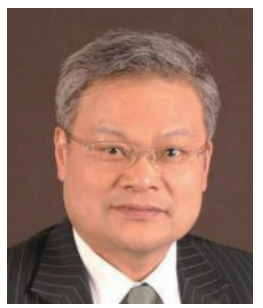

Jae One Lee received his B.E. and M.E. degrees from Dong-A University, Busan, Korea, in 1985 and 1988, respectively, and his Dr.-Ing. degree from Hannover University, Germany, in 1996. From 2000 to 2005, he worked for the Korean Association of Surveying and Mapping as a chief researcher. Since 2006, he has been a professor at Dong-A University, Korea. His research interests are in drone surveying, camera calibration, and cadastral surveying. (leejo@dau.ac.kr) 